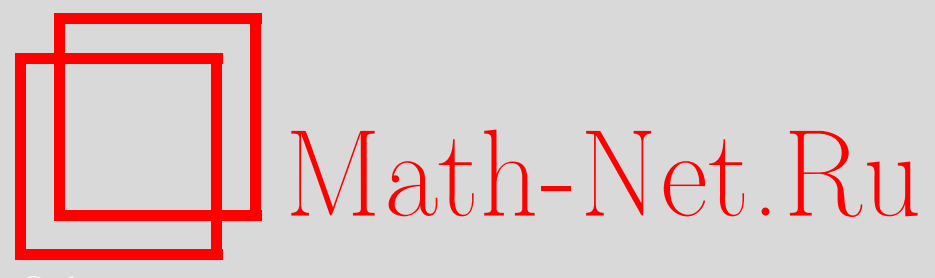

Д. А. Ошмарин, Н. В. Севодина, М. А. Юрлов, Н. А. Юрлова, Вариант мультимодального демпфирования колебаний электроупругих конструкций за счет соответствующего подбора параметров внешней электрической цепи, Вестн. Сам. гос. техн. ун-та. Сер. Физ.-мат. науки, 2016, номер 3, 475-495

DOI: https://doi.org/10.14498/vsgtu1509

Использование Общероссийского математического портала MathNet.Ru подразумевает, что вы прочитали и согласны с пользовательским соглашением

http://www . mathnet.ru/rus/agreement

Параметры загрузки:

IP : 34.227 .88 .159

26 апреля 2023 г., 15:12:00

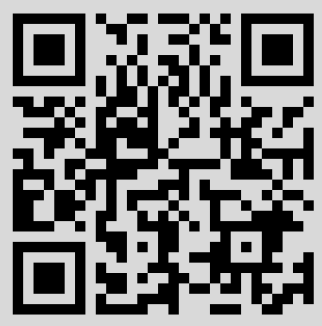




\title{
ВАРИАНТ МУЛЬТИМОДАЛЬНОГО ДЕМПФИРОВАНИЯ КОЛЕБАНИЙ ЭЛЕКТРОУПРУГИХ КОНСТРУКЦИЙ ЗА СЧЕТ СООТВЕТСТВУЮЩЕГО ПОДБОРА ПАРАМЕТРОВ ВНЕШНЕЙ ЭЛЕКТРИЧЕСКОЙ ЦЕПИ
}

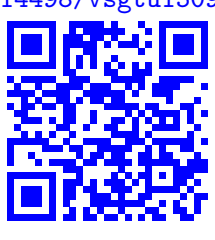

\section{Д. А. Оимарин, Н. В. Севодина, М. А. Юрлов, Н. А. Юрлова}

Институт механики сплошных сред УрО РАН, Россия, 614013, Пермь, ул. Акад. Королёва, 1.

\begin{abstract}
Аннотация
В технических приложениях имеет место задача демпфирования колебаний в заданных зонах конструкций, например, в местах расположения оптических приборов, при любых внешних динамических возмущениях, без увеличения массы самой конструкции и без изменения ее спектрального портрета. Для решения этих задач широкое распространение получило применение особых демпфирующих устройств - пьезоэлементов, соединенных с внешней электрической цепью и присоединенных к конструкции. Это стало возможным благодаря пьезоэффекту, обеспечивающему преобразование части энергии колебаний в электрическую энергию, которая рассеивается во внешней электрической цепи. Поэтому, применяя соответствующие электрические цепи, можно рассеять внутреннюю энергию и, как следствие, демпфировать колебания конструкции в определенном частотном диапазоне. Как правило, внешняя электрическая цепь с одной веткой, шунтирующая один пьезоэлемент, позволяет осуществить демпфирование колебаний на одной частоте. В связи с тем, что на практике часто возникает необходимость демпфирования нескольких мод колебаний с использованием одних и тех же технических средств, проблема мультимодального демпфирования smart-конструкций является актуальной. Целью данной работы является исследование возможностей демпфирования колебаний на нескольких модах с помощью одной внешней последовательной $R L$-цепи, присоединенной к электродированным поверхностям одного пьезоэлемента, на основе решения задач о собственных и вынужденных колебаниях электроупругих систем с внешними электрическими цепями.
\end{abstract}

(C) 2016 Самарский государственный технический университет.

\section{Образец для цитирования}

Ош м арин Д. А., Севодина Н. В., Юрлов М. А., Юрлова Н. А. Вариант мультимодального демпфирования колебаний электроупругих конструкций за счет соответствующего подбора параметров внешней электрической цепи // Becmн. Сам. гос. техн. ун-та. Сер. Физ.-мат. науки, 2016. Т. 20, № 3. С. 475-495. doi: 10.14498/vsgtu1509.

\section{Сведения об авторах}

Дмитрий Александрович Оимарин (oshmarin@icmm.ru; автор, ведущий переписку), аспирант, отд. комплексных проблем механики деформируемых твердых тел.

Наталья Виталвевна Севодина (к.т.н.; natsev@icmm.ru), научный сотрудник, отд. комплексных проблем механики деформируемых твердых тел.

Максим Александрович Юрлов (yurlovm@icmm.ru), инженер-исследователь, отд. комплексных проблем механики деформируемых твердых тел.

Наталия Алексеевна Юрлова (к.т.н., доцент; yurlova@icmm.ru), старший научный сотрудник, отд. комплексных проблем механики деформируемых твердых тел. 
Ключевые слова: электровязкоупругие системы с пьезоэлементами, внешние пассивные электрические цепи, мультимодальное демпфирование, собственные колебания, вынужденные колебания.

Введение. Скрепленные с механической конструкцией пьезоэлектрические элементы, к которым присоединены внешние электрические цепи, шунтирующие пьезоэлементы, образуют устройства, на которых происходит диссипация энергии и тем самым осуществляется дополнительное демпфирование колебаний. Пьезоэлектрический эффект обеспечивает преобразование части механической энергии колебаний в электрическую энергию, которая может быть рассеяна через шунтирующую цепь, представляющую собой механизм пассивного демпфирования. Поэтому, применяя соответствующие электрические цепи, можно рассеять внутреннюю энергию и, как следствие, подавить колебания посредством добавления пассивного демпфирования [1].

Ключевой проблемой демпфирования колебаний с помощью шунтирующих внешних электрических цепей является нахождение самой простой шунтирующей цепи, с помощью которой наиболее эффективно осуществляется демпфирование колебаний в требуемом диапазоне частот для конкретной конструкции. Требования к дизайну шунтирующей цепи могут быть сведены к следующим: шунтирующая цепь должна демпфировать механические колебания максимально эффективно, при этом ее стабильность должна быть гарантированной, не должна требовать для работы источник питания, стоимость исполнения и вес шунтирующей цепи должны быть сведены к минимуму; так как цепь интегрируется в конструкцию, то размер ее компонент должен быть минимальным [2].

За последнее десятилетие было разработано множество различных пассивных шунтирующих цепей, которые могут быть, согласно классификации, приведенной в [2], линейными и нелинейными. Среди линейных пассивных цепей, в свою очередь, можно выделить резистивные (состоящие только из резистора $R$ ) и резонансные (имеющие в своем составе сопротивление $R$ и индуктивность $L$ ) цепи.

Использование пьезоэлементов, зашунтированных резистивными цепями, приводит к тому, что динамическое поведение конструкции становится таким же, как при замене упругого материала конструкции вязкоупругим. Применение же резонансных $R L$-цепей, состоящих из последовательно либо параллельно соединенных катушки индуктивности и сопротивления, приводит к образованию колебательного контура, колебания которого обусловлены взаимодействием катушки индуктивности и собственной емкости пьезоэлемента, что приводит к возникновению в спектре частот колебаний системы (конструкции с пьезоэлементом и внешней электрической цепью) дополнительной резонансной частоты.

Путем изменения характеристик элементов внешней электрической цепи можно производить настройку этого дополнительного резонанса на конкретную частоту, совпадающую с собственной частотой колебаний конструкции, обеспечивая отвод энергии колебаний во внешнюю электрическую цепь, и тем самым эффективно подавлять колебания системы на данной частоте. В литературе динамическое поведение системы с внешней резонансной цепью часто сравнивают с поведением системы с динамическим демпфером [1]. 
В отличие от резистивных цепей, позволяющих снизить амплитуду колебаний на нескольких частотах, $R L$-цепи демпфируют только одну моду колебаний, так как совместно с пьезоэлементом образуют только один колебательный контур, частоту которого можно настроить на соответствующую частоту конструкции. Особенностью такого рода систем управления колебаниями является гарантируемая стабильность в присутствии конструкционных неопределенностей. Это качество обеспечивается фундаментальными свойствами пассивных систем демпфирования шунтированием [1-3].

Однако все резонансные шунты имеют общий недостаток-очень чувствительную к изменениям параметров системы степень демпфирования колебаний. При изменении внешнего воздействия на систему или при изменении параметров самой системы (вследствие, например, появления повреждений), меняется спектральный портрет системы, частота, на которую была настроена $R L$-цепь, меняется, а это, в свою очередь, приводит к тому, что внешняя электрическая цепь перестает эффективно работать. Попытки использования несколько отдельных $R L$-цепей не улучшают ситуацию, так как часто сбивается настройка отдельных шунтов, и они также перестают эффективно работать.

Поэтому развитие способов мультимодального (на нескольких частотах) демпфирования колебаний конструкции является актуальным. Сегодня можно условно выделить следующие основные группы подходов к мультимодальному демпфированию:

- использование одного пьезоэлемента и сложной внешней электрической цепи, в которой тем или иным способом реализована возможность демпфирования нескольких мод колебаний [4-12];

- применение нескольких пьезоэлементов, образующих единую сеть, соединенных тем или иным способом с одной внешней электрической цепью [13-17];

- использование нескольких пьезоэлементов, имеющих каждый свою отдельную электрическую цепь, которые в единую не объединяются [18, 19].

Каждый из этих подходов имеет свои достоинства и недостатки. Так, например, в случае сложных разветвленных цепей существенно возрастает сложность их настройки, поскольку каждая ветвь шунтирующей цепи не может рассматриваться как отдельный независимый шунт [4]. В этом случае можно использовать блокирующие либо проводящие цепи, подключенные последовательно к шунтирующим ветвям [5,8], что позволит рассматривать их как независимые. Однако при технической реализации это приводит к очень громоздким электрическим схемам за счет применения большого числа индуктивных элементов, что неизбежно сказывается на массовых характеристиках демпфирующих устройств. Использование электронных аналогов индуктивных элементов в какой-то мере помогает обойти данную проблему, но в силу того, что они являются лишь приближенным представлением идеальных индуктивных элементов, это не устраняет проблемы сложности настройки и подбора параметров цепи [10].

Использование нескольких пьезоэлементов с присоединенными к ним простыми $R L$-цепями дает возможность обойти проблему сложности настройки цепей, поскольку в этом случае каждый пьезоэлемент с присоединенной це- 
пью представляет собой изолированную систему, что позволяет подбирать параметры шунтирующих цепей независимо друг от друга. Тем не менее в [18] показано, что в случае мультимодального демпфирования данный подход не всегда эффективен, и все же рекомендуется решать оптимизационную задачу для поиска оптимальных значений параметров внешних цепей. Однако использование большого числа пьезоэлементов может привести к существенному увеличению массы конструкции и крайне негативно сказаться на ее динамических характеристиках. Таким образом, сегодня ряд проблем, связанных с применением пьезоэлементов и внешних электрических цепей для демпфирования нескольких мод колебаний, требует дальнейшего исследования и разработки новых подходов к реализации мультимодального демпфирования. В данной работе исследована возможность использования одного пьезоэлемента и одной внешней последовательной $R L$-цепи для демпфирования колебаний на нескольких частотах за счет соответствующего подбора параметров внешней электрической цепи.

1. Математическая постановка задачи. Рассматривается кусочно-однородное тело объемом $V=V_{1} \cup V_{2}$, где объем $V_{1}$ состоит из однородных упругих частей, а объем $V_{2}$ - из пьезоэлектрических элементов. Часть поверхности объема $V_{2}$ электродирована. K одной из электродированных поверхностей присоединена внешняя пассивная электрическая цепь произвольной конфигурации, состоящая из резистивных элементов (резисторов) сопротивлением $R$, индуктивных элементов (катушек индуктивности) с индуктивностью $L$ и емкостных элементов (конденсаторов) емкостью $C$.

Вариационное уравнение движения тела, состоящего из упругого и пьезоэлектрических элементов, формулируется на основе соотношений линейной теории упругости и квазистатических уравнений Максвелла [20-24]:

$$
\begin{aligned}
& \int_{V_{1}}\left(\sigma_{i j} \delta \varepsilon_{i j}+\rho \ddot{u}_{i} \delta u_{i}\right) d V+\int_{V_{2}}\left(\sigma_{i j} \delta \varepsilon_{i j}-D_{i} \delta E_{i}+\rho \ddot{u}_{i} \delta u_{i}\right) d V+ \\
& +\int_{\Gamma_{\sigma}}\left(\sigma_{i j} n_{j}\right) \delta u_{i} d S+\int_{\Omega_{e l}}\left(D_{i} n_{i}\right) \delta \varphi d S+\sum_{i=1}^{n_{L}} \frac{1}{L_{i}} \iint\left(\varphi_{1}^{L_{i}}-\varphi_{2}^{L_{i}}\right) \delta \varphi d t d t+ \\
& +\sum_{j=1}^{n_{R}} \frac{1}{R_{j}} \int\left(\varphi_{1}^{R_{j}}-\varphi_{2}^{R_{j}}\right) \delta \varphi d t+\sum_{k=1}^{n_{C}} C_{k}\left(\varphi_{1}^{C_{k}}-\varphi_{2}^{C_{k}}\right) \delta \varphi=0
\end{aligned}
$$

Здесь $\sigma_{i j}$ - компоненты симметричного тензора напряжений Коши, $\varepsilon_{i j}$ - компоненты тензора линейных деформаций, $u_{i}$ - компоненты вектора перемещений, $\rho$ - плотность материала, $D_{i}, E_{i}$ - компоненты векторов электрической индукции и напряженности электрического поля; $\varphi$ - электрический потенциал, $\varphi_{1}^{L_{i}}, \varphi_{2}^{L_{i}}, \varphi_{1}^{R_{j}}, \varphi_{2}^{R_{j}}, \varphi_{1}^{C_{k}} ; \varphi_{2}^{C_{k}}$ - значения потенциалов, соответственно, на элементах индуктивности, сопротивления и емкости; $L_{i}\left(i=1, \ldots, n_{L}\right)-$ величина индуктивности $i$-того индуктивного элемента; $R_{i}\left(i=1, \ldots, n_{R}\right)-$ величина сопротивления $i$-того индуктивного элемента; $C_{i}\left(i=1, \ldots, n_{C}\right)-$ величина емкости $i$-того индуктивного элемента; $n_{L}, n_{R}, n_{C}$ - количество индуктивных, резистивных и емкостных элементов соответственно; $\Omega_{e l}$ - часть поверхности пьезоэлектрического тела, покрытая электродами (электродированная поверхность); $n_{i}$ - координаты единичной нормали к электродиро- 
ванной поверхности. Для электрического поля выполняется условие потенциальности:

$$
\varphi,,_{i}=-E_{i}
$$

Для упругих частей объема $V_{1}$ выполняются физические уравнения линейной теории упругости:

$$
\sigma_{i j}=C_{i j k l} \varepsilon_{k l}
$$

При изотермических процессах электроупругие части объема $V_{2}$ удовлетворяют следующим физическим уравнениям:

$$
\left.\begin{array}{rl}
\sigma_{i j} & =\bar{C}_{i j k l} \varepsilon_{k l}-\beta_{i j k} E_{k}, \\
D_{k} & =\beta_{i j k} \varepsilon_{i j}+\epsilon_{k i} E_{i},
\end{array}\right\}
$$

где $C_{i j k l}, \bar{C}_{i j k l}$ - тензоры упругих констант, $\beta_{i j k}$ и $\epsilon_{k i}$ - тензоры пьезоэлектрических и диэлектрических коэффициентов.

На рассматриваемую систему накладываются ограничения в виде механических и электрических граничных условий. Механические граничные условия принимают следующий вид:

$$
\left.u_{i}\right|_{\Gamma_{u}}=u_{i 0},\left.\quad \sigma_{i j} n_{j}\right|_{\Gamma_{\sigma}}=p_{i}
$$

где $\Gamma_{u}$ - часть поверхности объема $V$, на которой заданы перемещения, $\Gamma_{\sigma}-$ часть поверхности объема $V$, на которой заданы внешние нагрузки. Выражения для электрических граничных условий для пьезоэлектрических частей, ограниченных объемом $V_{2}$, имеют следующий вид:

$$
\left.\varphi\right|_{\Omega_{e l}^{\varphi}}=\varphi_{0},\left.\quad D_{i} n_{i}\right|_{\Omega_{e l}^{Q}}=q_{s} .
$$

Здесь $\Omega_{e l}^{\varphi}$ - часть электродированной поверхности объема $V_{2}$, на которой задан электрический потенциал, $\Omega_{e l}^{Q}-$ часть электродированной поверхности объема $V_{2}$, на которой задана поверхностная плотность свободных зарядов $q_{s}$. В случае отсутствия электродирования все электрические граничные условия принимают нулевые значения.

2. Используемые методы и подходы. Как правило, при моделировании демпфирующие свойства smart-систем оцениваются по величине амплитуды при резонансном режиме или по скорости переходных процессов. В первом случае решается задача о вынужденных установившихся колебаниях, во втором - динамическая задача с начальными условиями. Приложения этих задач для поиска оптимальных параметров пьезоэлементов и внешних электрических цепей сопряжены с рядом проблем. В частности, для получения амплитуд при резонансных режимах на основе решения задачи о вынужденных установившихся колебаниях требуется многократное решение задачи при различных частотах внешних воздействий. При использовании задачи о вынужденных установившихся колебаниях или задачи с начальными условиями найденные оптимальные решения связаны с моделируемым вариантом нагружения исследуемой системы.

В работах [24-26] было показано, что для определения и оптимизации динамических характеристик системы, представляющей собой конструкцию, 
выполненную из упругого или вязкоупругого материала с присоединенными к ней пьезоэлементами и внешними электрическими цепями, наиболее эффективной является задача о собственных колебаниях. Это обусловлено тем, что получаемые в результате решения данной задачи динамические характеристики системы являются интегральными и не зависящими от вида и величины внешнего воздействия. При этом не требуется многократного повторения всех вычислительных процедур, связанных с изменением частотного диапазона и различными вариантами нагружения, как это имеет место при рассмотрении вынужденных установившихся колебаний или переходных процессов.

При рассмотрении собственных колебаний ставится задача поиска собственных решений для уравнения (1) вида

$$
u_{i}(x, t)=\bar{u}_{i}(\vec{x}) e^{i \omega t}, \quad \varphi(x, t)=\bar{\varphi}(\vec{x}) e^{i \omega t},
$$

где $\omega=\omega_{\mathrm{Re}}+i \omega_{\mathrm{Im}}-$ комплексная собственная частота колебаний. При этом $\omega_{\mathrm{Re}}$ соответствует собственной частоте, $\omega_{\mathrm{Im}}$ - скорости затухания колебаний, $\bar{u}_{i}(\vec{x}), \bar{\varphi}(\vec{x})$ - собственные формы колебаний. При решении задачи о собственных колебаниях интегралы в уравнении (1), соответствующие граничным условиям

$$
\int_{\Gamma_{\sigma}}\left(\sigma_{i j} n_{j}\right) \delta u_{i} d S, \quad \int_{\Omega_{e l}}\left(D_{i} n_{i}\right) \delta \varphi d S,
$$

принимают нулевые значения.

Для определения комплексных собственных частот электровязкоупругих систем с внешними пассивными электрическими цепями используется алгоритм, приведенный в работе [23].

3. Критерий для подбора параметров внешней электрической цепи, обеспечивающий демпфирование нескольких частот. При подборе параметров внешних цепей для демпфирования одной моды колебаний наиболее часто применяется условие минимума амплитуды колебаний на заданной частоте [1-3]. Однако в случае применения одной цепи для демпфирования нескольких частот данное условие нельзя использовать для формулировки критерия подбора параметров в силу того, что последовательная резонансная $R L$-цепь позволяет получить минимальное значение амплитуды только на одной конкретной частоте [1].

Вообще говоря, при реализации мультимодального демпфирования существует множество вариантов формулировки критерия оптимальности в зависимости от поставленной цели при решении той или иной задачи. Так, например, можно задать условие равенства амплитуд одновременно на нескольких частотах или установить предельное значение амплитуды, которое не должно превышаться на некотором частотном диапазоне, и т. д.

Поскольку динамические процессы, происходящие в рассматриваемой системе, исследуются на основе решений задачи о собственных колебаниях, для поиска критерия получения параметров внешней электрической цепи, обеспечивающих демпфирование нескольких мод колебаний, предлагается использовать мнимую часть комплексной собственной частоты. Этот подход можно считать оправданным исходя из практических соображений. Для реальных конструкций все колебательные процессы протекают во времени, и они далеко не всегда выходят на установившиеся режимы. И в этом случае важнейшую роль играет именно скорость затухания колебаний. 
Анализ поведения мнимых частей $\omega_{\text {Im }}$ комплексных собственных частот $\omega=\omega_{\operatorname{Re}}+i \omega_{\operatorname{Im}}$ в зависимости от изменения параметров сопротивления $R$ и индуктивности $L$ внешней последовательной $R L$-цепи показал, что для некоторых частот в пространстве параметров $R L$ существуют точки, в которых показатели демпфирования совпадают. На рис. 1 приведены примеры поверхности мнимых частей $\omega_{\text {Im }}$ для двух пар комплексных собственных частот в зависимости от сопротивления и индуктивности. На данных графиках четко видно линии пересечения, в которых мнимые части комплексных собственных частот совпадают.

На рис. 1, а видны два четких экстремума, которые соответствуют максимальной скорости затухания колебания для отдельных частот и реализуются при настройке внешней цепи на одну из этих частот. Здесь линия совпадения мнимых частей представляет собой гладкую пространственную кривую, на которой достаточно сложно выделить четкий экстремум.

На рис. $1, b$ приведено поведение другой пары мнимых частей комплексных собственных частот. Здесь на линии совпадения мнимых частей, в отличие от первого случая, можно заметить ярко выраженный экстремум, ко-

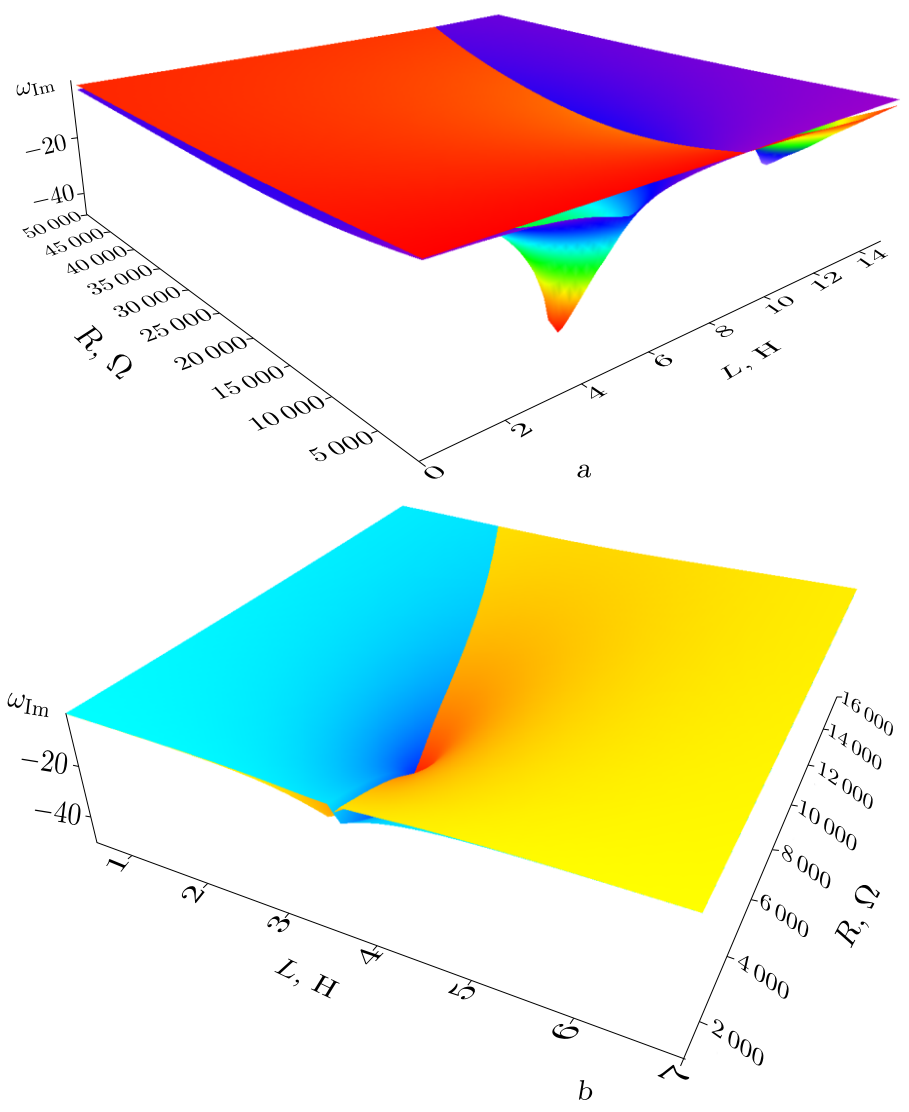

Рис. 1. Поверхности зависимости мнимых частей $\omega_{\text {Im }}$ двух различных пар комплексных собственных частот в пространстве параметров $R$ и $L$

[Figure 1. The relationship surface of imaginary parts $\omega_{\operatorname{Im}}$ for two different pairs of complex natural frequencies in the space of parameters $R$ and $L]$ 
торый позволяет подобрать параметры внешней цепи таким образом, чтобы скорость затухания колебаний была не просто одинаковой, но и максимальной для двух данных частот на линии равенства мнимых частей. Анализ поведения различных пар комплексных собственных частот в зависимости от изменения параметров сопротивления и индуктивности позволил сформулировать критерий для подбора параметров внешней последовательной $R L$-цепи, который обеспечит демпфирование колебаний для двух различных частот.

В качестве критерия для определения параметров внешней электрической цепи, при которых возможно демпфирование нескольких (пока попарно) мод колебаний, предлагается точка в пространстве параметров $R L$, отвечающая максимальному значению модуля мнимой части комплексной частоты на линии совпадения мнимых частей демпфируемых частот колебаний. Математически данный критерий можно сформулировать следующим образом

$$
\max \left(\left|\omega_{\operatorname{Im}}^{k}\right|=\left|\omega_{\operatorname{Im}}^{l}\right|\right),
$$

где $k, l$ - номера демпфируемых одновременно частот колебаний.

4. Результаты численных расчетов. Проиллюстрируем предлагаемый подход на результатах численных расчетов по определению оптимальных параметров внешней цепи для демпфирования колебаний на нескольких частотах. В качестве объектов исследования были выбраны консольнозащемленная пластинка и цилиндрическая оболочка, жестко защемленная по торцам и свободно опертая по образующим (рис. 2).

Размеры пластинки $210 \times 26 \times 0.5 \mathrm{мм}$, оболочки $-r=76 \mathrm{mм}, l=300 \mathrm{мм}$, $h=0.25$ мм. Для пластинки был выбран материал со следующими физикомеханическими характеристиками: $E=2 \cdot 10^{11} \Pi \mathrm{a}, \nu=0.3, \rho=7800 \mathrm{kг} / \mathrm{m}^{3}$. Физико-механические характеристики материала оболочки принимают следующие значения: $E=1.96 \cdot 10^{11} \Pi$ а, $\nu=0.3, \rho=7700 \mathrm{kг} / \mathrm{m}^{3}$.

Для пластинки пьезоэлемент выполнен в форме прямоугольника с размерами $50 \times 20 \times 0.36$ мм, для оболочки - в форме сегмента кольца со следующими размерами: $l_{p}=50 \mathrm{~mm}, \varphi=15.08^{\circ}, h=0.36$ мм. В качестве материала пьезоэлемента была выбрана пьезокерамика PZT-4 с поляризацией в направлении оси $z$ (пластинка) и оси $r$ (оболочка) и следующими физико-механи-

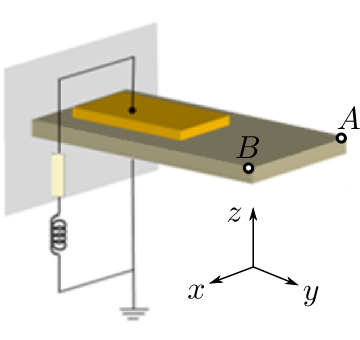

$a$

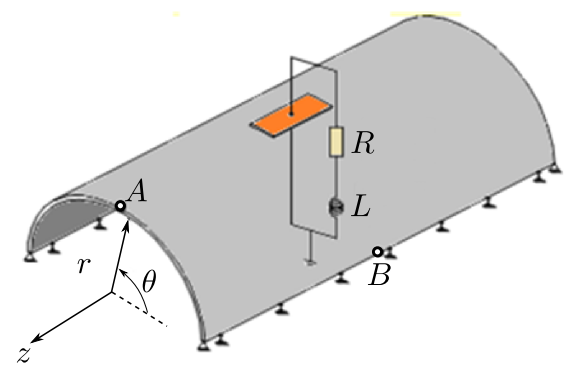

$b$

Рис. 2. Консольнозащемленная пластинка (а) и цилиндрическая оболочка, жестко защемленная по торцам и свободно опертая по образующим (b)

[Figure 2. Cantilever plate (a) and cylindrical shell, clamped on its ends and freely-supported on its edges $(b)]$ 
ческими характеристиками: $C_{11}=11.5 \cdot 10^{10}$ Па, $C_{12}=C_{13}=7.43 \cdot 10^{10}$ Па, $C_{23}=7.78 \cdot 10^{10} \Pi \mathrm{a}, C_{22}=C_{33}=13.9 \cdot 10^{10} \Pi \mathrm{a}, C_{44}=C_{66}=2.56 \cdot 10^{10}$ Па, $C_{55}=3.06 \cdot 10^{10} \Pi \mathrm{a}, \beta_{11}=15.2 \mathrm{Kл} / \mathrm{m}^{2}, \beta_{21}=\beta_{31}=-5.2 \mathrm{Kл} / \mathrm{m}^{2}, \beta_{42}=\beta_{63}=$ $=12.7 \mathrm{Kл} / \mathrm{M}^{2}, \epsilon_{11}=5.62 \cdot 10^{-9} \Phi / \mathrm{M}, \epsilon_{22}=\epsilon_{33}=6.45 \cdot 10^{-9} \Phi / \mathrm{M}, \rho=7500 \mathrm{K \Gamma} / \mathrm{m}^{3}$.

На первом этапе определялись спектры собственных частот колебаний конструкций с пьезоэлементом без внешней цепи на основе решения задачи о собственных колебаниях.

В табл. 1 приведены первые 10 собственных частот для пластинки с пьезоэлементом и первые 20 собственных частот для оболочки с пьезоэлементом.

Ранее отмечалось [26], что при конкретном размещении пьезоэлемента на упругом теле в спектре собственных частот колебаний присутствуют частоты, на которые внешняя электрическая цепь не оказывает никакого влияния при любых параметрах цепи. Данный эффект объясняется тем, что при колебаниях на этих частотах пьезоэлемент остается электрически нейтральным в силу того, что он либо не деформируется вообще, либо его деформация симметрична. Поэтому демпфировать колебания на этих частотах при данном положении пьезоэлемента невозможно. Для дальнейшего удобства вычислений и представлений результатов исключим их из рассмотрения (ячейки в табл. 1, в которых текст не выделен полужирным шрифтом) и пронумеруем подряд только рассматриваемые частоты (табл. 2).

Таблица 1

Собственные частоты колебаний конструкции с пьезоэлементом без внешней цепи [Natural vibration frequencies for structure with piezoelectric element and no external circuit]

\begin{tabular}{c|c|c|c|c|c}
\hline \multicolumn{2}{|c|}{$\begin{array}{c}\text { Пластинка с пьезоэлементом } \\
\text { [The plate with piezoelectric element] }\end{array}$} & \multicolumn{4}{|c}{$\begin{array}{c}\text { Оболочка с пьезоэлементом } \\
\text { [The shell with piezoelectric element] }\end{array}$} \\
\hline $\begin{array}{c}\text { Numbers of } \\
\text { frequencies }\end{array}$ & $f, \mathrm{~Hz}$ & $\begin{array}{c}\text { Numbers of } \\
\text { frequencies }\end{array}$ & $f, \mathrm{~Hz}$ & $\begin{array}{c}\text { Numbers of } \\
\text { frequencies }\end{array}$ & $f, \mathrm{~Hz}$ \\
\hline $\mathbf{1}$ & $\mathbf{1 3 . 8 6}$ & $\mathbf{1}$ & $\mathbf{5 5 7 . 4 1}$ & 11 & 1263.63 \\
$\mathbf{2}$ & $\mathbf{7 1 . 3 3}$ & 2 & 587.68 & $\mathbf{1 2}$ & $\mathbf{1 2 9 3 . 4 2}$ \\
$\mathbf{3}$ & $\mathbf{1 9 8 . 3 2}$ & 3 & 620.20 & 13 & 1325.30 \\
4 & 204.45 & $\mathbf{4}$ & $\mathbf{7 5 9 . 5 7}$ & 14 & 1430.59 \\
$\mathbf{5}$ & $\mathbf{4 0 4 . 1 5}$ & 6 & $\mathbf{8 0 3 . 7 9}$ & $\mathbf{1 5}$ & $\mathbf{1 4 8 2 . 2 1}$ \\
6 & 489.40 & 7 & 1012.10 & 17 & 1546.77 \\
7 & 584.10 & 8 & 1034.98 & 18 & 1598.97 \\
$\mathbf{8}$ & $\mathbf{6 7 4 . 9 6}$ & 9 & 1082.61 & 19 & 1594.05 \\
9 & 928.29 & 10 & 1161.89 & $\mathbf{2 0}$ & $\mathbf{1 6 4 1 . 6 4}$ \\
$\mathbf{1 0}$ & $\mathbf{9 9 7 . 5 4}$ & \multicolumn{4}{|c|}{} \\
\hline
\end{tabular}

Таблица 2

Собственные частоты колебаний конструкции с пьезоэлементом без внешней цепи, учитываемые в расчетах, после перенумерации [Natural vibration frequencies for structure with piezoelectric element and no external circuit taken into account after renumbering]

\begin{tabular}{c|c|c}
\hline Numbers of & The plate with piezoelectric element & The shell with piezoelectric element \\
\cline { 2 - 3 } frequencies & $f, \mathrm{~Hz}$ & $f, \mathrm{~Hz}$ \\
\hline 1 & 13.86 & 557.41 \\
2 & 72.33 & 587.68 \\
3 & 198.32 & 759.57 \\
4 & 404.15 & 803.79 \\
5 & 674.96 & 1482.21 \\
\hline
\end{tabular}


Рассмотрены два варианта внешних цепей: резистивная $R$-цепь и последовательная резонансная $R L$-цепь.

5.1. Резистивная $\boldsymbol{R}$-цепь. При подключении к пьезоэлементу внешней электрической цепи, состоящей из одного резистора (сопротивления), собственные частоты колебаний становятся комплексными $\left(\omega=\omega_{\operatorname{Re}}+i \omega_{\operatorname{Im}}\right)-$ появляется мнимая часть, или показатель демпфирования колебаний.

Влияние величины сопротивления $R$ на мнимую часть собственных частот колебаний пластинки (рис. 2) в соответствии с табл. 3 приведено на рис. 3 $(a-$ для второй и третьей частот, $b$ - для четвертой и пятой частот).

Для оболочки (рис. 2) значения первых пяти рассматриваемых собственных частот при различных значениях сопротивления $R$ приведены в табл. 4. Поведение мнимой части пяти полученных комплексных собственных частот колебаний оболочки в зависимости от величины сопротивления проиллюстрировано на рис. 4.

Таким образом, полученные результаты демонстрируют возможность снижения амплитуды колебаний всех или некоторых из определенного диапазона частот резонансов, если ввести в электрическую цепь резистор определенной величины сопротивления $R$.

Например, для рассматриваемой пластинки максимального снижения амплитуды колебаний на 2 и 3 резонансах с помощью пьезоэлемента и одной электрической цепи можно добиться при сопротивлении $R \sim 125 \mathrm{kOм}$, а на 4 и 5 резонансах - при сопротивлении $R \sim 23$ кОм; для оболочки же максимальное снижение амплитуды колебаний на первых пяти резонансных частотах обеспечит сопротивление во внешней электрической цепи в диапазоне от 10 до 20 кОм.

Таким образом, приведенное в [1] утверждение, что поведение конструкции из упругого материала при подключении к пьезоэлементу внешней элек-

\section{Таблица 3}

Значения комплексных собственных частот колебаний пластинки при различных значениях сопротивления $R$ [Complex natural vibration frequencies for the plate at different values of resistance $R$ ]

\begin{tabular}{c|c|c|c}
\hline Numbers of frequencies & $R=1 \mathrm{k} \Omega$ & $R=23 \mathrm{k} \Omega$ & $R=125 \mathrm{k} \Omega$ \\
\hline 1 & $13.46-0.0003 i$ & $13.46-0.006 i$ & $13.46-0.034$ \\
2 & $71.45-0.001 i$ & $71.46-0.02 i$ & $71.51-0.083 i$ \\
3 & $196.01-0.01 i$ & $196.05-0.12 i$ & $196.32-0.18 i$ \\
4 & $396.18-0.12 i$ & $397.44-1.97 i$ & $400.12-1.13 i$ \\
5 & $659.53-0.42 i$ & $664.39-4.76 i$ & $668.67-1.62 i$ \\
\hline
\end{tabular}

Таблица 4

Значения комплексных собственных частот колебаний оболочки при различных значениях сопротивления $R$ [Complex natural vibration frequencies for the shell at different values of resistance $R]$

\begin{tabular}{c|c|c|c}
\hline Numbers of frequencies & $R=1 \mathrm{k} \Omega$ & $R=10 \mathrm{k} \Omega$ & $R=20 \mathrm{k} \Omega$ \\
\hline 1 & $554.26-0.13 i$ & $554.70-1.11 i$ & $555.51-1.55 i$ \\
2 & $755.78-0.22 i$ & $756.71-1.66 i$ & $757.93-1.90 i$ \\
3 & $800.14-0.19 i$ & $800.90-1.51 i$ & $802.04-1.85 i$ \\
4 & $1287.70-0.55 i$ & $1290.40-2.94 i$ & $1292.20-2.43 i$ \\
5 & $1471.70-1.07 i$ & $1477.10-5.41 i$ & $1480.30-4.28 i$ \\
\hline
\end{tabular}




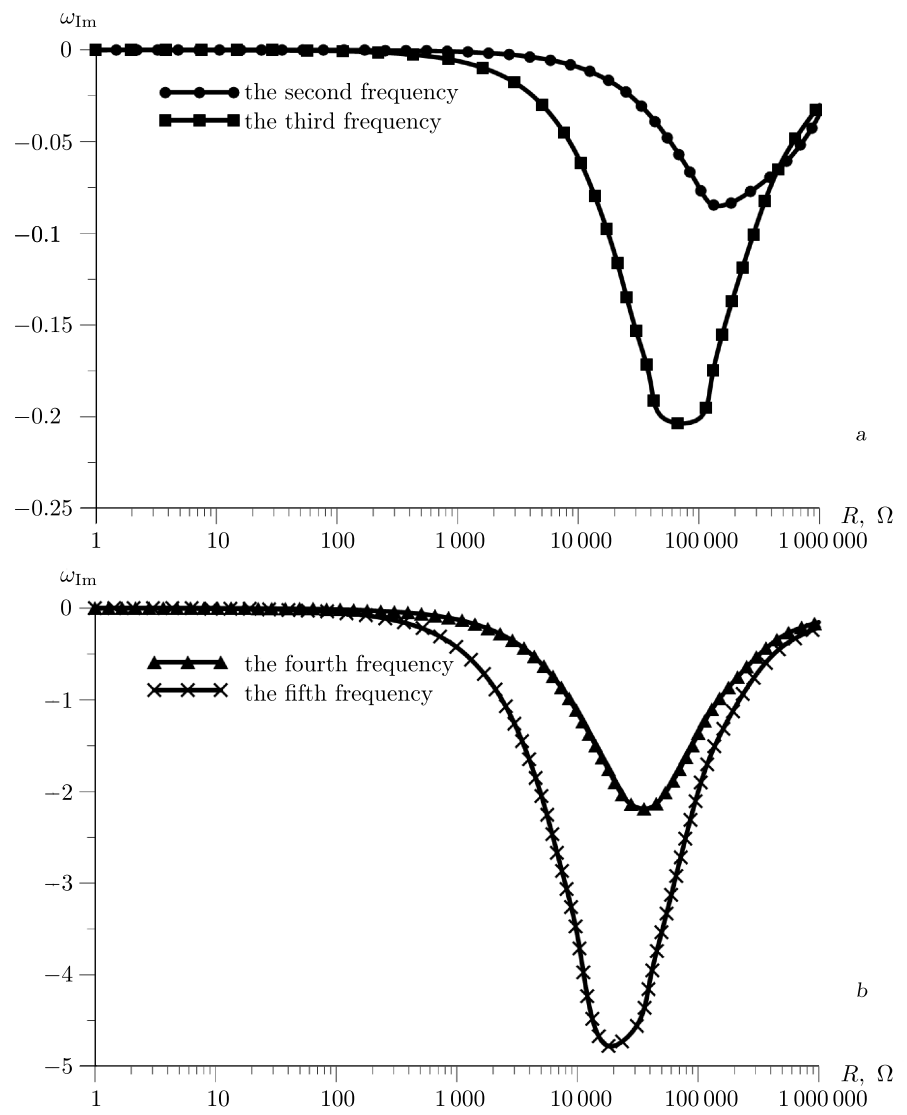

Рис. 3. Зависимость показателя демпфирования $\omega_{\operatorname{Im}}$ (мнимой части комплексной собственной частоты $\left.\omega=\omega_{\mathrm{Re}}+i \omega_{\mathrm{Im}}\right)$ от величины внешнего сопротивления для консольнозащемленной пластинки для второй и третьей частот (a), четвертой и пятой частот $(b)$

[Figure 3. Relationship between damping ratio $\omega_{\text {Im }}$ (imaginary part of complex natural frequency $\omega=\omega_{\operatorname{Re}}+i \omega_{\text {Im }}$ ) and external resistance magnitude for the cantilever plate for the second and third frequencies (a) and for the forth and fifth frequencies (b)]

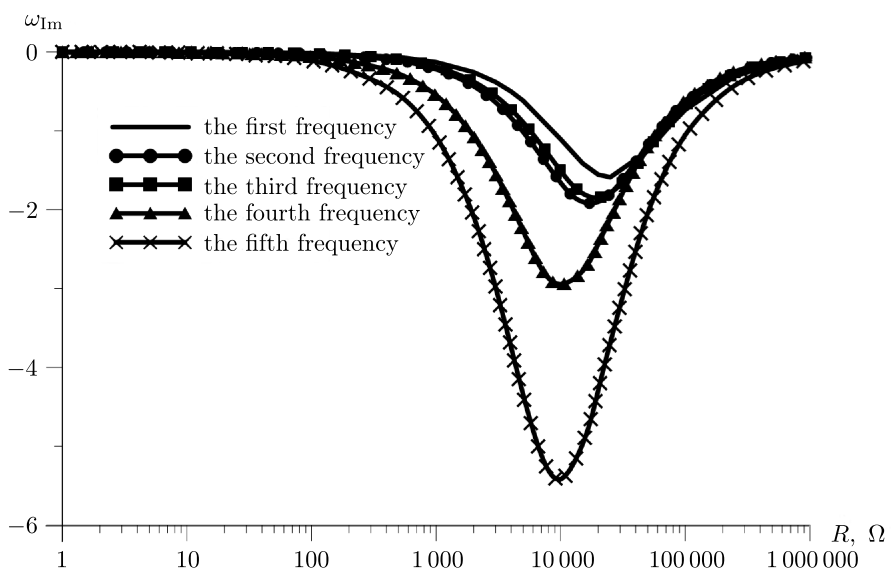

Рис. 4. Зависимость показателя демпфирования $\omega_{\operatorname{Im}}$ (мнимой части комплексной собственной частоты $\left.\omega=\omega_{\mathrm{Re}}+i \omega_{\mathrm{Im}}\right)$ от величины внешнего сопротивления для цилиндрической оболочки [Figure 4. Relationship between damping ratio $\omega_{\text {Im }}$ (imaginary part of complex natural frequency $\left.\omega=\omega_{\mathrm{Re}}+i \omega_{\mathrm{Im}}\right)$ and external resistance magnitude for the shell] 
трической цепи, состоящей из одного резистора, изменяется таким же образом, как при замене упругого материала конструкции вязкоупругим, подтверждено численно.

Кроме этого, численно показано, что эффект мультимодального демпфирования для упругой конструкции при присоединении к пьезоэлементу простой резистивной цепи получается естественно - в некотором диапазоне значений сопротивления происходит снижение амплитуды колебаний всех рассматриваемых частот колебаний.

5.2. Последовательная резонансная $\boldsymbol{R} \boldsymbol{L}$-цепь. Так как присоединение к пьезоэлементу, скрепленному с конструкцией, внешней резонансной $(R L)$ электрической цепи приводит к образованию колебательного контура, то путем подбора соответствующих параметров сопротивления и индуктивности он может быть «настроен» на одну из частот колебаний конструкции. В связи с этим становится привлекательной идея демпфирования одной внешней резонансной $R L$-цепью нескольких частот колебаний рассматриваемой конструкции с одним пьезоэлементом.

Сначала оценим возможность демпфирования двух частот колебаний. При этом ограничимся рассмотрением применения последовательной $R L$-цепи. Для рассматриваемых конструкций (пластинка и оболочка, см. рис. 2) проведены расчеты по определению параметров сопротивления $R$ и индуктивности $L$, при которых возможно демпфирование некоторых мод колебаний на основе предложенного критерия (2). Результаты расчетов приведены в табл. 5 (пластинка) и 6 (оболочка). Индексы у $R$ и $L$ обозначают номера демпфируемых частот.

Сравнение результатов, приведенных в табл. 3, 4, с результатами, приведенными в табл. 5, 6, приводит к выводу о том, что использование для демпфирования двух частот колебаний последовательной резонансной $R L$ цепи эффективнее, чем резистивной $R$-цепи, поскольку в данном случае показатели демпфирования (мнимые части собственных частот) существенно выше.

Анализируя данные табл. 6, можно заметить, что для всех пар демпфируемых частот значения оптимальных параметров индуктивности $L$ находятся очень близко, что может косвенно свидетельствовать о возможном существовании таких параметров $R$ и $L$, которые позволят демпфировать колебания оболочки на этих частотах.

В результате расчетов были подобраны такие пары параметров сопротивления и индуктивности, которые оказывают влияние на частоты со второй по пятую $\left(R_{2-5}, L_{2-5}\right)$ и с первой по пятую $\left(R_{1-5}, L_{1-5}\right)$. Здесь индексы у $R$ и $L$ обозначают диапазон рассматриваемых частот. В табл. 7 приведены значения данных пар параметров и соответствующие им значения комплексных собственных частот для оболочки.

В последних столбцах табл. 5 и 7 приведены значения максимально возможной величины коэффициента демпфирования (мнимой части комплексной частоты) той частоты колебаний, на демпфирование которой настраивается внешняя цепь подбором соответствующих величин сопротивления и индуктивности.

При подборе параметров внешней цепи, позволяющих демпфировать колебания на нескольких частотах, показатели демпфирования будут ниже, чем 


\section{Таблица 5}

Результаты расчетов оптимальных параметров последовательной $R L$-цепи для пластинки [The results of calculation of optimal parameters of series $R L$ circuit for the plate]

\begin{tabular}{l|c|l|l}
\hline $\begin{array}{l}\text { Номера } \\
\text { демпфи- } \\
\text { руемых } \\
\text { попарно } \\
\text { частот }\end{array}$ & $\begin{array}{c}\text { Оптимальные } \\
\text { значения } \\
\text { параметров } \\
R \text { (кОм), } L(\Gamma \text { н) }\end{array}$ & $\begin{array}{l}\text { Значения } \\
\text { комплексных } \\
\text { собственных } \\
\text { частот }\end{array}$ & $\begin{array}{l}\text { Максимально возможные значения } \\
\text { показателей демпфирования } \omega_{\text {Im при }}^{i} \\
\text { настройке внешней цепи на конкрет- } \\
\text { ную частоту }\end{array}$ \\
\hline \multirow{2}{*}{2 и 3} & $\begin{array}{l}R_{23}=126 \\
L_{23}=143.5\end{array}$ & $\begin{array}{c}72.19-0.14 i \\
198.44-0.14 i\end{array}$ \\
\hline \multirow{2}{*}{4 и 5} & $R_{45}=14.8$ & $398.67-6.12 i$ & 2.56 \\
& $L_{45}=9.1$ & $682.16-5.84 i$ & 29.45 \\
\hline
\end{tabular}

Таблица 6

Результаты расчетов оптимальных параметров последовательной $R L$-цепи для оболочки [The results of calculation of optimal parameters of series $R L$ circuit for the shell]

\begin{tabular}{|c|c|c|c|c|c|}
\hline $\begin{array}{l}\text { Номера } \\
\text { демпфи- } \\
\text { руемых } \\
\text { частот }\end{array}$ & $\begin{array}{c}\text { Оптимальные } \\
\text { значения } \\
\text { параметров } \\
R(\text { КОм }) \\
L\left(\Gamma_{\mathrm{H}}\right)\end{array}$ & $\begin{array}{l}\text { Значения } \\
\text { комплексных } \\
\text { собственных } \\
\text { частот }\end{array}$ & $\begin{array}{l}\text { Номера } \\
\text { демпфи- } \\
\text { руемых } \\
\text { частот }\end{array}$ & $\begin{array}{c}\text { Оптимальные } \\
\text { значения } \\
\text { параметров } \\
R(\text { кОм }) \\
L\left(\Gamma_{\mathrm{H}}\right) \\
\end{array}$ & $\begin{array}{l}\text { Значения } \\
\text { комплексных } \\
\text { собственных } \\
\text { частот }\end{array}$ \\
\hline 1 и 2 & $\begin{array}{l}R_{12}=8.4 \\
L_{12}=4.9\end{array}$ & $\begin{array}{l}552.57-5.12 i \\
763.68-4.78 i \\
\end{array}$ & 2 и 4 & $\begin{array}{l}R_{24}=7.5 \\
L_{24}=2.2 \\
\end{array}$ & $\begin{array}{l}755.01-4.81 i \\
1297.7-4.14 i\end{array}$ \\
\hline 1 и 3 & $\begin{array}{l}R_{13}=8.2 \\
L_{13}=5.0\end{array}$ & $\begin{array}{l}552.37-5.36 i \\
808.37-5.21 i \\
\end{array}$ & 2 и 4 & $\begin{array}{l}R_{25}=8.8 \\
L_{25}=2.3\end{array}$ & $\begin{array}{l}755.86-5.01 i \\
1488.25-4.28 i\end{array}$ \\
\hline 1 и 4 & $\begin{array}{c}R_{14}=15.1 \\
L_{14}=2.8\end{array}$ & $\begin{array}{l}554.81-2.64 i \\
1295.53-2.47 i \\
\end{array}$ & 3 и 4 & $\begin{array}{l}R_{34}=6.1 \\
L_{34}=2.3 \\
\end{array}$ & $\begin{array}{l}797.93-4.35 i \\
1298.4-3.53 i \\
\end{array}$ \\
\hline 1 и 5 & $\begin{array}{c}R_{15}=14.1 \\
L_{15}=3.1\end{array}$ & $\begin{array}{l}554.62-2.84 i \\
1486.04-2.62 i\end{array}$ & 3 и 5 & $\begin{array}{c}R_{35}=10.6 \\
L_{35}=2.4\end{array}$ & $\begin{array}{l}800.59-4.68 i \\
1487.38-4.09 i\end{array}$ \\
\hline 2 и 3 & $\begin{array}{l}R_{23}=4.0 \\
L_{23}=3.7\end{array}$ & $\begin{array}{l}777.37-18.54 i \\
814.11-28.55 i\end{array}$ & 4 и 5 & $\begin{array}{l}R_{45}=5.7 \\
L_{45}=2.3\end{array}$ & $\begin{array}{l}1298.62-3.44 i \\
1489.73-3.46 i\end{array}$ \\
\hline
\end{tabular}

Таблица 7

Результаты расчетов оптимальных параметров последовательной $R L$-цепи для оболочки [The results of calculation of optimal parameters of series $R L$ circuit for the shell]

\begin{tabular}{|c|c|c|c|}
\hline $\begin{array}{l}\text { Диапазон } \\
\text { демпфи- } \\
\text { руемых } \\
\text { частот } \\
(\text { с - по })\end{array}$ & $\begin{array}{c}\text { Оптимальные } \\
\text { значения } \\
\text { параметров } \\
R(\text { (КОм }), L(\Gamma н)\end{array}$ & $\begin{array}{l}\text { Значения } \\
\text { комплексных } \\
\text { собственных } \\
\text { частот }\end{array}$ & $\begin{array}{l}\text { Максимально возможные значения } \\
\text { показателей демпфирования } \omega_{\text {Im }}^{i} \text { при } \\
\text { настройке внешней цепи на конкрет- } \\
\text { ную частоту }\end{array}$ \\
\hline $1-5$ & $\begin{array}{l}R_{1-5}=7.5 \\
L_{1-5}=2.3\end{array}$ & $\begin{array}{l}553.51-1.75 i \\
755.02-5.14 i \\
798.88-4.58 i \\
1297.7-3.71 i \\
1488.9-4.03 i\end{array}$ & $\begin{array}{l}29.55 \\
37.86 \\
38.22 \\
61.05 \\
88.02\end{array}$ \\
\hline $2-5$ & $\begin{array}{c}R_{2-5}=10.0 \\
L_{2-5}=4.0\end{array}$ & $\begin{array}{l}761.15-6.61 i \\
804.89-7.33 i \\
1295.8-1.05 i \\
1485.8-1.22 i\end{array}$ & $\begin{array}{l}37.86 \\
38.22 \\
61.05 \\
88.02\end{array}$ \\
\hline
\end{tabular}


при настройке на демпфирование одной частоты. Но хотя при этом и снижается степень демпфирования колебаний, рабочий диапазон действия внешней электрической цепи расширяется, становится широкополосным. По значениям максимально возможной величины коэффициента демпфирования можно оценить степень мультимодального демпфирования (насколько меньше снижается амплитуда колебаний по сравнению с одномодовым демпфировани$\mathrm{em})$.

6. Задача о вынужденных установившихся колебаниях. Для демонстрации влияния параметров $R$ и $L$ внешней электрической цепи на динамическое поведение рассматриваемой системы была решена задача о вынужденных установившихся гармонических колебаниях.

Для пластинки (рис. 2) решалась задача при различных значениях параметров внешних цепей, в том числе и подобранных таким образом, чтобы демпфировать две соседние частоты.

Амплитудно-частотная характеристика перемещений

$$
U_{\text {sum }}=\sqrt{U_{x}^{2}+U_{y}^{2}+U_{z}^{2}}
$$

точки $A$ системы (рис. 2, а) строилась от воздействия внешних осевых гармонических сил с амплитудой $P_{x}=P_{y}=P_{z}=1 \mathrm{H}$, приложенных в угловой точке $B$ на незакрепленной грани пластины.

Проведено сравнение амплитуд перемещений при следующих значениях параметров $R$ и $L: R=1$ кОм (принято произвольно), а $R=23$ кОм и $R=125$ кОм подобраны таким образом, что при резистивной цепи обеспечивают максимальные показатели демпфирования попарно 4-5 и 2-3 частот колебаний. $R_{23}, L_{23}$ и $R_{45}, L_{45}$ - подобранные пары параметров для демпфирования попарно 2-3 и 4-5 частот колебаний при резонансной внешней электрической цепи соответственно. Индексы у $R$ и $L$ обозначают номера частот, демпфирование которых возможно при данных параметрах.

На первом графике (рис. 5, а) видно, что при значениях компонент электрической цепи $R_{23}$ и $L_{23}$, подобранных для демпфирования второй и третьей частот, достигается наилучшее соотношение по величине амплитуд перемещений колебаний пластинки на данных частотах. При этом для второй частоты значение амплитуды перемещений по сравнению с другими вариантами $R$ и $L$ будет минимальным.

Второй график (рис. 5, b) приведен для четвертой и пятой частот. Видно, что подобранные для этих частот параметры внешней цепи $R_{45}, L_{45}$ также обеспечивают наилучшее демпфирование колебаний на этих частотах. При этом установлено, что внешняя электрическая цепь с параметрами, позволяющими демпфировать колебания на 4 и 5 резонансных частотах, существенно снижает величину амплитуды колебаний системы и на предыдущей, третьей частоте.

Аналогичным образом было оценено влияние подобранных параметров внешней электрической цепи $R$ и $L$ на примере расчета вынужденных гармонических колебаний цилиндрической оболочки (рис. $2, b)$.

Амплитудно-частотные характеристики строились в точке $A$ оболочки с пьезоэлементом и с внешней последовательной $R L$-цепью от воздействия гармонической силы с амплитудой $P_{r}=1 \mathrm{H}$, приложенной в точке $B$. 
В ходе расчетов было установлено, что при использовании пары $L_{2-5}=$ $=2.3 \Gamma \mathrm{H}, R_{2-5}=7.5$ кОм существенно снижается амплитуда колебаний на частотах со второй по пятую, а при $L_{1-5}=4.0$ Гн, $R_{1-5}=10 \mathrm{kOм}-$ уже с первой по пятую.

Сравнение амплитуд колебаний проведено при различных значениях параметров $R L: R=1$ кОм (принято произвольно), а $R=10$ кОм и $R=20$ кОм подобраны таким образом, чтобы обеспечить максимальные показатели демп-

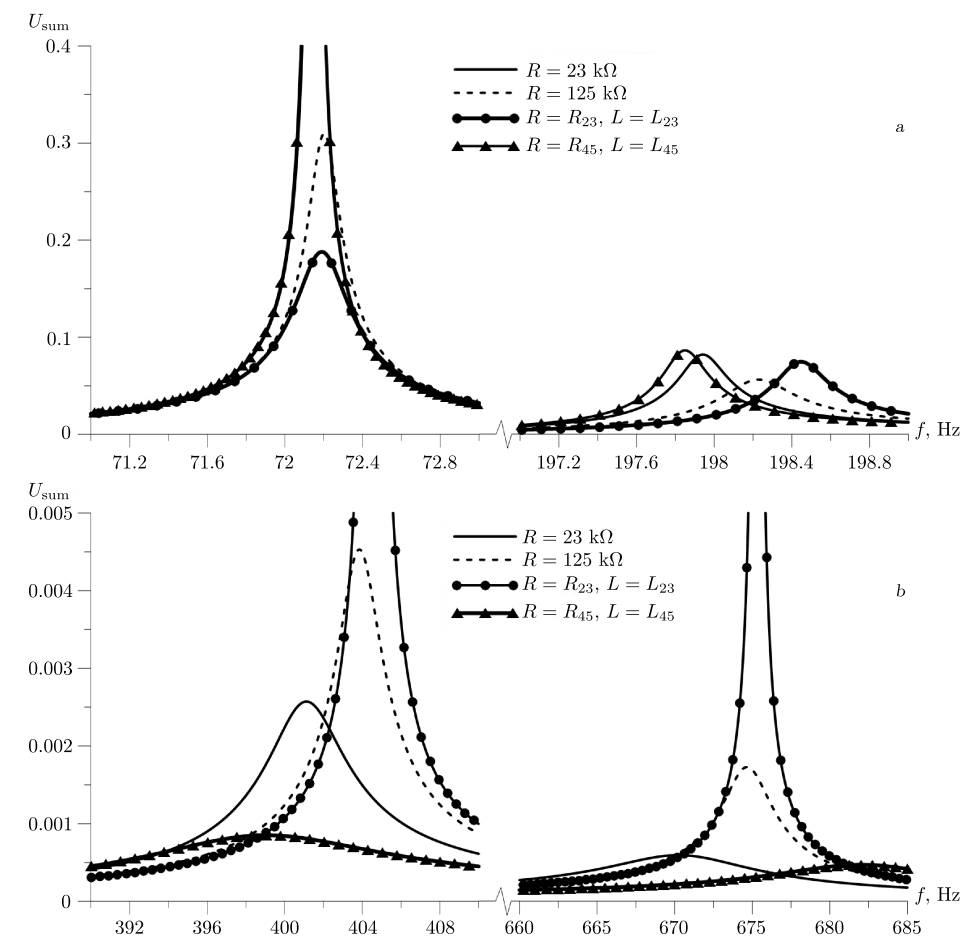

Рис. 5. Амплитудно-частотные характеристики второй и третьей частот (a) и четвертой и пятой частот $(b)$ пластинки при различных значениях параметров внешних цепей

[Figure 5. Amplitude vs. frequency plots for the second and third (a) and the forth and fifth frequencies $(b)$ at different values of external circuit parameters]

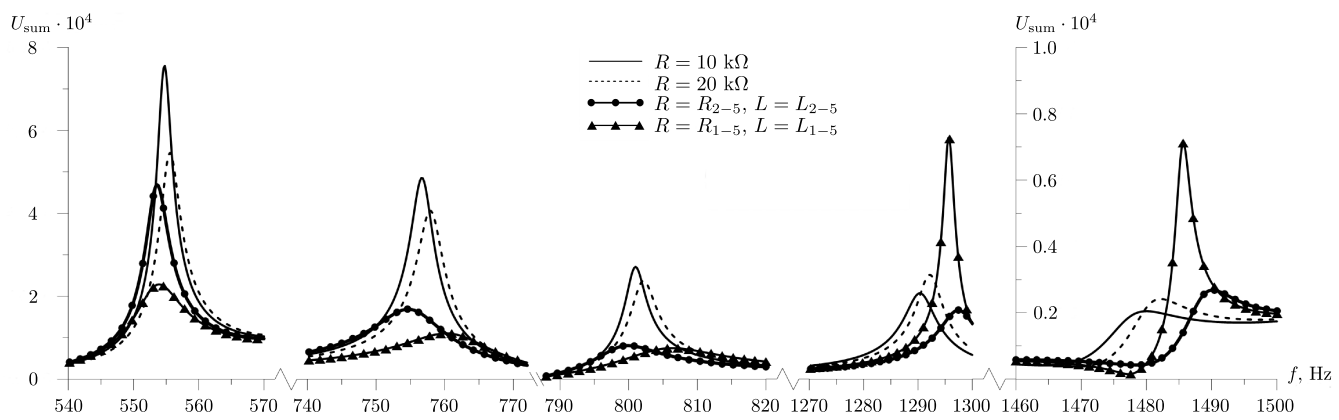

Рис. 6. Амплитудно-частотные характеристики для первых пяти частот колебаний оболочки при различных значениях параметров внешних цепей

[Figure 6. Amplitude vs. frequency plots for the first five vibrational frequencies for the shell at different values of external circuit parameters] 
фирования у 4-5 и с первой по третью частот колебаний при резистивной цепи, $R_{2-5}, L_{2-5}$ и $R_{1-5}, L_{1-5}$ - подобранные пары параметров компонент электрической цепи, позволяющие демпфировать колебания на резонансных частотах со 2 по 5 и с 1 по 5 соответственно. Индексы у $R$ и $L$ обозначают диапазон частот: $1-5-$ с первой по пятую, 2-5- со второй по пятую. Амплитудно-частотные характеристики для первых пяти частот колебаний оболочки при различных значениях параметров внешних цепей приведены на рис. 6. Видно, что оба варианта настройки, с первой по пятую и со второй по пятую, дают существенное снижение амплитуды колебаний для всех пяти частот.

При этом обнаружено, что вариант с параметрами $R_{2-5}, L_{2-5}$ предпочтителен, так как при этом максимальное значение амплитуды колебаний оболочки в рассматриваемом диапазоне частот, в данном случае реализующееся на первой частоте, меньше, чем максимальное значение амплитуды при применении параметров $R_{1-5}, L_{1-5}$, реализующееся при этом на четвертой частоте.

Выводы. Проведено исследование возможности использования для демпфирования колебаний на нескольких частотах (мультимодальное демпфирование) одного пьезоэлемента, соединенного с одной резистивной или последовательной резонансной $R L$-цепью.

Предложен критерий для определения параметров внешней последовательной резонансной $R L$-цепи, обеспечивающих демпфирование колебаний на двух и более частотах.

Показано, что подобранные на основе предложенного критерия пары параметров резонансной $R L$-цепи дают большее снижение амплитуд колебаний, чем при наличии просто резистивной цепи, и позволяют демпфировать колебания на нескольких частотах, обеспечивая тем самым мультимодальное демпфирование.

Декларация о финансовых и других взаимоотношениях. Работа выполнена при поддержке Российского фонда фундаментальных исследований (проект № 16-31-00094-мол_а). Все авторы принимали участие в разработке концепции статьи и в написании рукописи. Авторы несут полную ответственность за предоставление окончательной рукописи в печать. Окончательная версия рукописи была одобрена всеми авторами. Авторы не получали гонорар за статью.

\footnotetext{
ORCIDs

Дмитрий Александрович Ошмарин: http://orcid.org/0000-0002-9898-4823

Наталья Витальевна Севодина: http://orcid.org/0000-0001-9374-7135

Максим Александрович Юрлов: http://orcid.org/0000-0003-1507-6617

Наталия Алексеевна Юрлова: http://orcid.org/0000-0003-3497-0358
}

\section{БИБЛИОГРАФИЧЕСКИЙ СПИСОК}

1. Hagood N. W., von Flotow A. Damping of structural vibrations with piezoelectricmaterials and passive electrical networks // Journal of Sound and Vibration, 1991. vol. 146, no. 2. pp. 243-268. doi : 10.1016/0022-460X (91) 90762-9.

2. Moheimani S. O. R., Fleming A. J. Piezoelectric transducers for vibration control and damping/ Advances in Industrial Control. London: Springer-Verlag, 2006. xvi+271 pp. doi: $10.1007 / 1-84628-332-9$

3. Wu S. Y. Piezoelectric shunts with parallel $R-L$ circuit for structural damping and vibration control// Proc. SPIE, 1996. vol. 2720, Smart Structures and Materials 1996: Passive Damping and Isolation. pp. 259-269. doi : 10.1117/12.239093.

4. Hollkamp J. J. Multimodal passive vibration suppression with piezoelectric materials and 
resonant shunts // Journal of Intelligent Material Systems and Structures, 1996. vol. 5, no. 1. pp. 49-57. doi: 10.1177/1045389x9400500106.

5. Wu S. Y. Method for multiple mode shunt damping of structural vibration using a single PZT transducer // Proc. SPIE, 1998. vol. 3327, Smart Structures and Materials 1998: Passive Damping and Isolation. pp. 159-168. doi: 10.1117/12.310680.

6. Wu S. Y. Multiple PZT transducers implemented with multiple-mode piezoelectric shunting for passive vibration damping // Proc. SPIE, 1999. vol. 3672, Smart Structures and Materials 1999: Passive Damping and Isolation. pp. 112-122. doi: 10.1117/12.349774.

7. Wu S. Y., Bicos A. S. Structural vibration damping experiments using improved piezoelectric shunts // Proc. SPIE, 1997. vol.3045, Smart Structures and Materials 1997: Passive Damping and Isolation. pp. 40-50. doi: 10.1117/12.274217.

8. Behrens S., Moheimani S. O. R. Current flowing multiple mode piezoelectric shunt dampener // Proc. SPIE, 2002. vol.4697, Smart Structures and Materials 2002: Damping and Isolation. pp. 117-127. doi : 10.1117/12.472658.

9. Behrens S., Moheimani S. O. R., Fleming A. J. Multiple mode passive piezoelectric shunt dampener / Proc. IFAC Mechatronics (Berkeley, CA, Dec. 2002), 2002.

10. Fleming A. J., Moheimani S. O. R. Adaptive piezoelectric shunt damping // Smart Materials and Structures, 2003. vol. 12, no. 1. pp. 36-48. doi: 10.1088/0964-1726/12/1/305.

11. Behrens S., Moheimani S. O. R. Optimal resistive elements for multiple mode shunt damping of a piezoelectric laminate beam / Proceedings of the 39th IEEE Conference on Decision and Control. vol. 4, 2000. pp. 4018-4023. doi: 10.1109/CDC.2000.912343.

12. Tai-Hong Cheng, Il-Kwon Oh A current-flowing electromagnetic shunt damper for multimode vibration control of cantilever beams // Smart Materials and Structures, 2009. vol. 18, no. 9, 095036. doi: 10.1088/0964-1726/18/9/095036.

13. Vidoli S., dell'Isola F. Vibration control in plates by uniformly distributed PZT actuators interconnected via electric networks // European Journal of Mechanics - A/Solids, 2001. vol. 20, no. 3. pp. 435-456. doi : 10.1016/S0997-7538(01)01144-5.

14. Porfiri M., dell'Isola F., Mascioli F. M. Circuit analog of a beam and its application to multimodal vibration damping, using piezoelectric transducers // International Journal of Circuit Theory and Applications, 2004. vol.32, no.4. pp. 167-198. doi: 10.1002/cta. 273.

15. dell'Isola F., Henneke E. G., Porfiri M. Piezoelectromechanical structures: new trends towards the multimodal passive vibration control // Proc. SPIE, 2003. vol. 5052, Smart Structures and Materials 2003: Damping and Isolation. pp. 392-402. doi: 10.1117/12.483803.

16. Maurini C., dell'Isola F., Del Vescovo D. Comparison of piezoelectronic networks acting as distributed vibration absorbers // Mechanical Systems and Signal Processing, 2004. vol. 18, no. 5. pp. 1243-1271. doi : 10.1016/S0888-3270(03)00082-7.

17. Giorgio I., Culla A., Del Vescovo D. Multimode vibration control using several piezoelectric transducers shunted with a multiterminal network// Arch. Appl. Mech, 2009. vol. 79, no. 9. pp. 859-879. doi: 10.1007/s00419-008-0258-x.

18. Viana F. A. C., Steffen V., Jr. Multimodal Vibration Damping through Piezoelectric Patches and Optimal Resonant Shunt Circuits // J. Braz. Soc. Mech. Sci. ES Eng., 2006. vol. 28, no. 3. pp. 293-310. doi : 10.1590/S1678-58782006000300007.

19. Casadei F., Ruzenne M., Dozio L., Cunefare K. A. Broadband vibration control through periodic arrays of resonant shunts: experimental investigation on plates // Smart Materials and Structures, 2010. vol. 19, no. 1, 015002. doi: 10.1088/0964-1726/19/1/015002.

20. Васидзу К. Вариационные методы в теории упругости и пластичности. М.: Мир, 1987. $542 \mathrm{c}$.

21. Партон В. З., Кудрявцев Б. А. Электромагнитоупругость пъезоэлектрических и электропроводных тел. М.: Наука, 1988. 471 с.

22. Карнаухов В. Г., Киричок И. Ф. Электротермовязкоупругость. Киев: Наук. думка, 1988. 319 c.

23. Матвеенко В. П., Клигман Е. П., Юрлова Н. А., Юрлов М. А. Моделирование и оптимизация динамических характеристик smart-структур с пьезоматериалами // Физическая мезомеханика, 2012. Т. 15, № 1. С. 75-85. 
24. Matveenko V. P., Yurlov M. A., Yurlova N. A. Optimization of the damping properties electro-viscoelastic objects with external electric circuits/ Mechanics of $A d$ vanced Materials: Analysis of Properties and Performance; eds. V. V. Silberschmidt, V. P. Matveenko. Switzerland: Springer International Publishing, 2015. pp. 79-100. doi : 10. 1007/978-3-319-17118-0_4.

25. Sevodina N. V., Yurlova N. A., Oshmarin D. A. The optimal placement of the piezoelectric element in a structure based on the solution of the problem of natural vibrations // Solid State Phenomena, 2016. vol.243. pp. 67-74. doi: 10.4028/www.scientific.net/ssp. 243. 67.

26. Иванов А. С., Матвеенко В. П., Ошмарин Д. А., Севодина Н. В., Юрлов М. А., Юрлова Н. А. Обоснование использования эквивалентных схем замещения для оптимизации диссипативных свойств электроупругих тел с внешними электрическими цепями // Изв. РАН. МТТ, 2016. № 3. С. 273-283.

Поступила в редакцию 13/VII/2016;

в окончательном варианте - 14/VIII/2016;

принята в печать - 09/IX/2016. 
Vestn. Samar. Gos. Techn. Un-ta. Ser. Fiz.-mat. nauki

[J. Samara State Tech. Univ., Ser. Phys. \& Math. Sci.], 2016, vol. 20, no. 3, pp. $475-495$

ISSN: 2310-7081 (online), 1991-8615 (print)

doi: http://dx.doi.org/10.14498/vsgtu1509

MSC: 74M05, 74F15, 74H45

\title{
VARIANT OF MULTIMODAL VIBRATION DAMPING OF ELECTROVISCOELASTIC STRUCTURES BY APPROPRIATE CHOICE OF EXTERNAL ELECTRIC CIRCUIT PARAMETERS
}

\author{
D. A. Oshmarin, N. V. Sevodina, M. A. Yurlov, N. A. Yurlova \\ Institute of Continuous Media Mechanics, Ural Branch of RAS, \\ 1, Akad. Korolyova st., Perm, 614013, Russian Federation.
}

\begin{abstract}
In technical applications it takes place the problem of vibration damping in certain regions of the structure, at the location of optical sensors for instance, at any external dynamic excitations with no mass increase and no changes in spectral portrait. In order to solve these problems it is widespread the use of special damping devices: piezoelectric elements connected to external electric circuits and attached to the structure. It became possible due to piezoelectric effect, which provides transformation of part of energy of vibrations into electric one, which is dissipated in external electric circuit. So that by using appropriate electric circuits one may dissipate internal energy and therefore reduce structural vibrations in definite frequency range. As a rule, external circuit of single branch, which shunts single piezoelectric element, allows vibration damping on one certain frequency. Due to the fact, that practical applications usually include requirements of damping of several modes by one and the same technical devices, the problem of multimodal vibration damping in smart-structures is rather acute. The objective of this paper is the study of possibility of vibration damping on several modes by using single external series $R L$-circuit, connected to electrodes of single piezoelectric element on the basis of solution of problems on natural and forced steady-state vibrations of electroelastic systems with external electric circuits.
\end{abstract}

Keywords: electroviscoelastic structures with piezoelectric elements, passive external electric circuits, multimodal damping, natural vibrations, forced vibrations.

(C) 2016 Samara State Technical University.

Please cite this article in press as:

Oshmarin D. A., Sevodina N. V., Yurlov M. A., Yurlova N. A. Variant of multimodal vibration damping of electroviscoelastic structures by appropriate choice of external electric circuit parameters, Vestn. Samar. Gos. Tekhn. Univ., Ser. Fiz.-Mat. Nauki [J. Samara State Tech. Univ., Ser. Phys. \& Math. Sci.], 2016, vol. 20, no. 3, pp. 475-495. doi: 10.14498/vsgtu1509. (In Russian)

\section{Authors Details:}

Dmitrii A. Oshmarin (oshmarin@icmm.ru; Corresponding Author), Postgraduate Student, Dept. of complex problems in solid mechanics.

Natalya V. Sevodina (Cand Techn. Sci.; natsev@icmm.ru), Researcher, Dept. of complex problems in solid mechanics.

Maksim A. Yurlov (yurlovm@icmm.ru), Research Engineer, Dept. of complex problems in solid mechanics.

Natalya A. Yurlova (Cand. Phys. \& Math. Sci.; yurlova@icmm.ru), Senior Researcher, Dept. of complex problems in solid mechanics. 
Declaration of Financial and Other Relationships. This work was supported by the Russian Foundation for Basic Research (project no. 16-31-00094-mol_a). Each author has participated in the article concept development and in the manuscript writing. The authors are absolutely responsible for submitting the final manuscript in print. Each author has approved the final version of manuscript. The authors have not received any fee for the article.

\author{
ORCIDs \\ Dmitrii A. Oshmarin: http://orcid.org/0000-0002-9898-4823 \\ Natalya V. Sevodina: http://orcid.org/0000-0001-9374-7135 \\ Maksim A. Yurlov: http://orcid.org/0000-0003-1507-6617 \\ Natalya A. Yurlova: http://orcid.org/0000-0003-3497-0358
}

\title{
REFERENCES
}

1. Hagood N. W., von Flotow A. Damping of structural vibrations with piezoelectricmaterials and passive electrical networks, Journal of Sound and Vibration, 1991, vol.146, no. 2, pp. 243-268. doi : 10.1016/0022-460X (91)90762-9.

2. Moheimani S. O. R., Fleming A. J. Piezoelectric transducers for vibration control and damping, Advances in Industrial Control. London, Springer-Verlag, 2006, xvi +271 pp. doi : 10.1007/1-84628-332-9

3. Wu S. Y. Piezoelectric shunts with parallel $R-L$ circuit for structural damping and vibration control, Proc. SPIE, 1996, vol. 2720, Smart Structures and Materials 1996: Passive Damping and Isolation, pp. 259-269. doi : 10.1117/12.239093.

4. Hollkamp J. J. Multimodal passive vibration suppression with piezoelectric materials and resonant shunts, Journal of Intelligent Material Systems and Structures, 1996, vol. 5, no. 1, pp. 49-57. doi : 10.1177/1045389X9400500106.

5. Wu S. Y. Method for multiple mode shunt damping of structural vibration using a single PZT transducer, Proc. SPIE, 1998, vol. 3327, Smart Structures and Materials 1998: Passive Damping and Isolation, pp. 159-168. doi: 10.1117/12.310680.

6. Wu S. Y. Multiple PZT transducers implemented with multiple-mode piezoelectric shunting for passive vibration damping, Proc. SPIE, 1999, vol. 3672, Smart Structures and Materials 1999: Passive Damping and Isolation, pp. 112-122. doi: 10.1117/12.349774.

7. Wu S. Y., Bicos A. S. Structural vibration damping experiments using improved piezoelectric shunts, Proc. SPIE, 1997, vol. 3045, Smart Structures and Materials 1997: Passive Damping and Isolation, pp. 40-50. doi: 10.1117/12.274217.

8. Behrens S., Moheimani S. O. R. Current flowing multiple mode piezoelectric shunt dampener, Proc. SPIE, 2002, vol. 4697, Smart Structures and Materials 2002: Damping and Isolation, pp. 117-127. doi : 10.1117/12.472658.

9. Behrens S., Moheimani S. O. R., Fleming A. J. Multiple mode passive piezoelectric shunt dampener, Proc. IFAC Mechatronics (Berkeley, CA, Dec. 2002), 2002.

10. Fleming A. J., Moheimani S. O. R. Adaptive piezoelectric shunt damping, Smart Materials and Structures, 2003, vol. 12, no. 1, pp. 36-48. doi : 10.1088/0964-1726/12/1/305.

11. Behrens S., Moheimani S. O. R. Optimal resistive elements for multiple mode shunt damping of a piezoelectric laminate beam, Proceedings of the 39th IEEE Conference on Decision and Control, vol. 4, 2000, pp. 4018-4023. doi: 10.1109/CDC.2000.912343.

12. Tai-Hong Cheng, Il-Kwon Oh A current-flowing electromagnetic shunt damper for multimode vibration control of cantilever beams, Smart Materials and Structures, 2009, vol. 18, no. 9, 095036. doi : 10.1088/0964-1726/18/9/095036.

13. Vidoli S., dell'Isola F. Vibration control in plates by uniformly distributed PZT actuators interconnected via electric networks, European Journal of Mechanics - A/Solids, 2001, vol. 20, no. 3, pp. 435-456. doi : 10.1016/S0997-7538(01)01144-5.

14. Porfiri M., dell'Isola F., Mascioli F. M. Circuit analog of a beam and its application to multimodal vibration damping, using piezoelectric transducers, International Journal of Circuit Theory and Applications, 2004, vol.32, no.4, pp. 167-198. doi: 10.1002/cta.273. 
15. dell'Isola F., Henneke E. G., Porfiri M. Piezoelectromechanical structures: new trends towards the multimodal passive vibration control, Proc. SPIE, 2003, vol. 5052, Smart Structures and Materials 2003: Damping and Isolation, pp. 392-402. doi: 10.1117/12.483803.

16. Maurini C., dell'Isola F., Del Vescovo D. Comparison of piezoelectronic networks acting as distributed vibration absorbers, Mechanical Systems and Signal Processing, 2004, vol.18, no. 5, pp. 1243-1271. doi: 10.1016/S0888-3270(03)00082-7.

17. Giorgio I., Culla A., Del Vescovo D. Multimode vibration control using several piezoelectric transducers shunted with a multiterminal network, Arch. Appl. Mech, 2009, vol.79, no. 9, pp. 859-879. doi : 10.1007/s00419-008-0258-x.

18. Viana F. A. C., Steffen V., Jr. Multimodal Vibration Damping through Piezoelectric Patches and Optimal Resonant Shunt Circuits, J. Braz. Soc. Mech. Sci. ES Eng., 2006, vol. 28, no. 3, pp. 293-310. doi: 10.1590/S1678-58782006000300007.

19. Casadei F., Ruzenne M., Dozio L., Cunefare K. A. Broadband vibration control through periodic arrays of resonant shunts: experimental investigation on plates, Smart Materials and Structures, 2010, vol. 19, no. 1, 015002. doi : 10.1088/0964-1726/19/1/015002.

20. Washizu K. Variational Methods in Elasticity and Plasticity. London, Pergamon Press, 1982, $540 \mathrm{pp}$.

21. Parton V. Z., Kudryavtsev B. A. Electromagnetoelasticity: Piezoelectrics and Electrically Conductive Solids. New York, Gordon and Breach Science Publ. Ltd, 1988, 503 pp.

22. Karnaukhov V. G., Kirichok I. F. Elektrotermoviazkouprugost' [Electrothermal viscoelasticity]. Kiev, Nauk. dumka, 1988, 319 pp. (In Russian)

23. Matveenko V. P., Kligman E. P., Yurlov M. A., Yurlova N. A. Simulation and optimization of dynamic characteristics of piezoelectric smart structures, Phys. Mesomech., 2012, vol. 15, no. 3, pp. 190-199. doi: 10.1134/S1029959912020063.

24. Matveenko V. P., Yurlov M. A., Yurlova N. A. Optimization of the damping properties electro-viscoelastic objects with external electric circuits, Mechanics of Advanced Materials: Analysis of Properties and Performance; eds. V. V. Silberschmidt, V. P. Matveenko. Switzerland, Springer International Publishing, 2015, pp. 79-100. doi : 10. 1007/978-3-319-17118-0_4.

25. Sevodina N. V., Yurlova N. A., Oshmarin D. A. The optimal placement of the piezoelectric element in a structure based on the solution of the problem of natural vibrations, Solid State Phenomena, 2016, vol. 243, pp. 67-74. doi: 10.4028/www.scientific.net/ssp.243.67.

26. Ivanov A. S. Matvienko V. P. Oshmarin D. A, Sevodina N. V., Yurlov M. A., Yurlova N. A. Justification of equivalent substitution circuits used to optimize the dissipative properties of electroelastic bodies with external electric circuits, Mechanics of Solids, 2016, vol.51, no. 3, pp. 273-283. doi: 10.3103/S0025654416030043.

Received 13/VII/2016;

received in revised form 14/VIII/2016;

accepted 09/IX/2016. 